the specimens were injected with colored latex while the PSTJs were respectively injected with $1 \mathrm{ml}$ of latex of a contrasting color under US guidance. Following the injections, the joints were frozen and cut into 2 centimeter sections from medial to lateral in the sagittal plane. Sections were examined independently by three authors for the presence of latex within the ASTJ, PSTJ and, also, to look for the presence of extra-articular latex extravasation or the spread of latex to adjacent articulations by means of a communication with the ankle joint or the adjacent portion of the subtalar joint.

Results: Six cryopreserved intact ankle-foot specimens from three male and three female cadavers (two right and four left), with a mean age of 74 years (range, 66-80 years) were studied. A recommended list of standardized scanning technique which allows sonographers to evaluate both compartments of the subtalar joint (ASTJ and PSTJ) from the medial, lateral and posterior aspect were developed. All of the specimens $(6 / 6,100 \%)$ contained the appropriate colored latex in the appropriate subtalar joint compartment with minimal leakage into the surrounding soft tissues (Figure 1$)$. Of note, five of the six $(5 / 6,83 \%)$ specimens revealed communication between the PSTJ and the posterior tibiotalar joint. There was no communication between the ASTJ and the tibiotalar joint, nor was there evidence of spread of the latex, i.e. communication, from one subtalar joint compartment to the other.

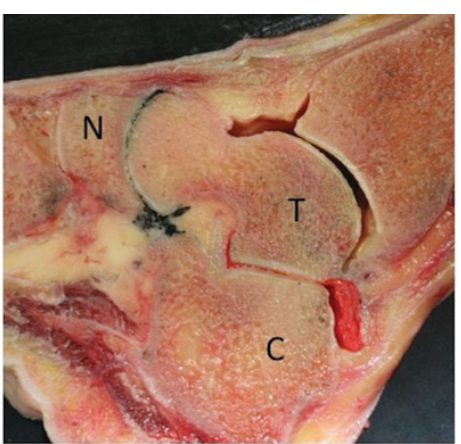

Figure 1. Anatomical specimen showing the anterior subtalar joint (filled with green latex) anc the posterior subtalar joint (filled with red latex);

$\mathrm{C}$ : calcaneus, $\mathrm{N}$ : navicular, $\mathrm{T}$ : talus

Conclusions: Lack of communication between the ASTJ and the PSTJ and the communication between the PSTJ and the posterior recess of the tibiotalar joint are compatible with other studies. Our study highlights the importance of employing cadaver specimens in musculoskeletal ultrasound, and presents a number of options for US imaging of both the ASTJ and PSTJ that also provide access to these distinct compartments for the purpose of aspiration and injection.

Disclosure of Interest: None declared

DOI: 10.1136/annrheumdis-2017-eular.2675

\section{FRI0669 VALIDITY OF SEVEN-JOINT VERSUS SIMPLIFIED TWELVE-JOINT ULTRASONOGRAPHY SCORING SYSTEMS IN ASSESSMENT OF RHEUMATOID ARTHRITIS ACTIVITY}

R. El-Gohary ${ }^{1}$, A. Ahmed ${ }^{1}$, A. Khalil ${ }^{2}$, H. El-Gendy ${ }^{1}$, K. Gado ${ }^{1}$. ${ }^{1}$ Internal Medicine, Rheumatology \& Clinical Immunology Subspeciality, Kasr Alainy; ${ }^{2} \mathrm{New}$ Kasr El-Aini Teaching Hospital, Cairo, Egypt

Background: Musculoskeletal ultrasonography imposes itself as reliable tool for the disease activity assessment of rheumatoid arthritis (RA) being more sensitive than clinical in detection of synovitis. There is no consensus on the exact joint number should be examined. Naredo et al. developed reduced 12-joint count ${ }^{1}$ while Backhaus et al. scored only seven small joints ${ }^{2}$. Although both were found to reflect disease activity it is suitable in a busy clinic to assess the lowest joint count.

Objectives: To investigate the validity of 7-joint ultrasonography (US7) scores in assessment of disease activity in Egyptian RA patients compared with simplified 12-joint ultrasonography (US12) scores and correlate both to composite disease activity indices.

Methods: Fifty Egyptian RA adult patients diagnosed according to the ACR1987 criteria were subjected to detailed history, 28 tender \& swollen joint counts (TJC, SJC). The disease activity was assessed by calculating DAS28-CRP, SDAI and CDAI. The Ultrasonographic assessment was performed using a LOGIQP6 with 10-13 MHz broadband linear array transducer by one well-trained blinded rheumatologist. The synovial hypertrophy on GS \& PD images were graded using a semi-quantitative $0-3$ scale. The simplified US12 was performed as originally described ${ }^{1}$. However the GS \& PD synovitis were computed in two separate scores instead of one. The US7 was performed at the clinically dominant side as described by Backhaus et $\mathrm{al}^{2}$. Two Sum-US7 scores were added; Sum (GS)-US7 after grading the GS-tenosynovitis and Sum (PD)-US7. Ultrasonography inter/intraobserver reliability was evaluated on 40 stored images using randomization and blinded techniques. The study was approved by the local ethics committee.

Results: The studied population had mean disease duration of $89 \pm 60$ months.
$62 \%$ were using more than one DMARD. They showed moderate disease activity as defined by DAS28, SDAI and CDAI (mean 3.7 $\pm 1.4,19.9 \pm 14.9$ \& $17.9 \pm 13.3$ respectively). The GS-scores showed no correlation with disease activity parameters. PD-US12, PD (synovitis)-US7 and Sum (PD)-US7 significantly correlated with the SJC, CRP levels (P values $0.03,0.04$ and 0.01$)$ and DAS28 (P values $0.02,0.009$ and 0.001 ), furthermore Sum (PD)-US7 correlated with SDAI and CDAI ( $P$ values $0.013 \& 0.042$ respectively). Correlation coefficient between the different ultrasonographic scores showed no differance. The ultrasonography reliability showed significant agreement $(P<0.001)$. Kappa values were 0.80 \& 0.96 for GS \& PD interobserver reliability and 0.96 \& 1.0 for GS \& PD intraobserver reliability.

Conclusions: Our results confirm the validity of PD-scores in assessment of disease activity. However they make the significance of GS questionable. The Sum-(PD) US7 seems to perform better than the original one, it was strongly correlated with CRP and with the three disease composite indices. The studied scores correlated with each other favoring the use of less time consuming US7score.

[1] Naredo E, et al. Validity, reproducibility, and responsiveness of a twelve-joint simplified power Doppler ultrasonographic assessment of joint inflammation in rheumatoid arthritis. Arthritis Care Res. 2008;59(4):515-22.

[2] Backhaus $M$, et al. Evaluation of a novel 7-joint ultrasound score in daily rheumatologic practice: A pilot project. Arthritis Care Res. 2009;61(9):1194201.

Disclosure of Interest: None declared

DOI: 10.1136/annrheumdis-2017-eular.3256

\section{FRI0670 THE COURSE OF SUBCLINICAL JOINT INFLAMMATION DURING PROGRESSION FROM ARTHRALGIA TO CLINICALLY DETECTABLE ARTHRITIS, A LONGITUDINAL MRI STUDY}

R.M. Ten Brinck, H.W. van Steenbergen, L. Mangnus, A.H. van der Helm-van Mil. Rheumatology, Leiden University Medical Centre, Leiden, Netherlands

Background: Subclinical joint inflammation in the phase of arthralgia preceding RA can comprise synovitis, bone marrow edema (BME) and/or tenosynovitis. It is unknown in what time order the different tissues in a joint become inflamed or progress with inflammation. It has been postulated that synovitis is an initial process that is succeeded by bone involvement ("outside-in hypothesis" presuming that synovitis precedes BME and erosions). Alternatively, it is suggested that inflammatory cells in the bone marrow migrate via bone pores to the synovium, promoting synovitis ("inside-out", then BME precedes synovitis). Thirdly, these processes can occur or progress simultaneously. Serial MRI studies can reveal time-relationships. This longitudinal MRI study on patient-level determined the course of joint inflammation during progression from arthralgia to clinical arthritis. Objectives: To increase the comprehension on the course of MRI-detected subclinical joint inflammation during progression from arthralgia to clinical arthritis, both in ACPA-positive and ACPA-negative arthritis patients.

Methods: We longitudinally followed 29 patients that all progressed from Clinically Suspect Arthralgia to clinical arthritis. 1.5T MRI on hand and foot joints was performed at presentation with arthralgia and subsequently at the development of clinical arthritis. MRIs were evaluated for BME, synovitis and tenosynovitis (summed in the total inflammation score) and erosions by three readers (ICCs $0.98,0.96$ and 0.97 ) that were blind to clinical data and the order in time. Analyses were repeated in arthritis patients that fulfilled the 2010 criteria for rheumatoid arthritis, and in ACPA-positive and ACPA-negative patients separately.

Results: At presentation with arthralgia the mean age was 43 years, $72 \%$ was female, and $28 \%$ was ACPA-positive. Median duration to clinical arthritis was 17 weeks. At the time of arthritis development $65 \%$ fulfilled the 2010 -criteria. During progression from arthralgia to clinical arthritis the median total inflammation score increased from 4.5 (IQR 2.8-7.8) to 6.0 (IQR 2.0-13.5), $\mathrm{p}=0.01$. The BME score increased over time $(p=0.04)$, as did the synovitis score $(p=0.002)$. The tenosynovitis and erosion scores increased as well, though not statistically significant $(p=0.10$ and $p=0.07$ respectively). Analyses within the patients that developed 2010-RA revealed that BME $(p=0.047)$, synovitis $(p=0.005)$ and tenosynovitis $(p=0.004)$ all increased significantly, in contrast to the erosion score. At presentation with arthralgia, BME and synovitis scores were higher in ACPA-positive than in ACPA-negative patients; during progression to clinical arthritis the different types of inflammation increased similarly in ACPA-positive and ACPA-negative patients.

Conclusions: During progression from arthralgia to clinically evident rheumatoid arthritis, BME, synovitis as well as tenosynovitis progress simultaneously.

Disclosure of Interest: None declared

DOI: 10.1136/annrheumdis-2017-eular.2908 